\title{
SOBRE LAS INSTITUCIONES PÚBLICAS COMO INSTRUMENTOS FEMINISTAS EMANCIPADORES PARA UN MUNDO EN TRANSICIÓN
}

\author{
LAURA GÓMEZ \\ Ex-Directora de igualdad de la DFG y técnica de igualdad local
}

DOI: $10.1387 /$ lan-harremanak.16100

\section{ABSTRACT}

En este artículo se propone analizar algunos de los desafios que deben ser afrontados si queremos imaginar hacer de las instituciones públicas instrumentos feministas al servicio de transitar hacia un horizonte común cuyo fin último sea sostener la vida humana y no humana en términos de universalidad. Y se propone hacerlo a partir de lo soñado y practicado bajo el impulso de la Dirección de igualdad (DI) de la Diputación Foral de Gipuzkoa (DFG) del gobierno de Bildu (2011-2015). Para ello, en primer lugar, estudiaremos el contexto en el que se enmarca la llegada de Bildu al gobierno de la DFG y el marco conceptual que sostiene la propuesta política que se hizo desde la DI. A continuación, se analizan algunas de las claves para la reinvención feminista de las instituciones. Y finalmente, se extraen algunas lecciones que quieren servir de faro en la consolidación de los procesos de tránsito iniciados desde estas.

Palabras clave: sostenibilidad de la vida, pedagogía, instituciones públicas, transición, feminismo.

This article aims at analysing some of the challenges that have to be faced if we want to turn public institutions into tools for a feminist transition towards a common future that sustains human life and our environment from a universal right's 
perspective. We will base our analysis on the policies that were imagined and implemented by the Direction for Gender Equality (DGE) of the Provincial Government of Gipuzkoa, in the Basque Country (Diputación Foral de Gipuzkoa, DFG), during the four-year term of the left-nationalist coalition named Bildu (2011-2015). Firstly, we will describe the background situation that led to Bildu's arrival to power, before outlining the conceptual framework that sets the base for the political proposal designed by the DGE. Secondly, we will analyse some of the key factors that are necessary for a feminist reinventing of the public institutions. Finally, we will draw a few lessons from the experience, in order to show the way towards the consolidation of the transitional processes that are initiated by such institutions.

Keywords: sustanibility of life, pedagogy, public institutions, transition, feminism.

Artikulu honen helburua aurre egin behar zaien erronka batzuk aztertzea da, erakunde publikoak, abiapuntu komun baterantz joateko zerbitzura dauden tresna feministak bihurtzea imajinatu nahi badugu, zeinen azken helburua unibertsaltasun terminoetan giza- eta ez giza-bizitza eustea den. Eta horrela egiten du Gipuzkoako Foru Aldundiko (GFA) Bilduren gobernuaren (2011-2015) Berdintasunerako Zuzendaritzaren (BZ) gidaritzapean amestu eta praktikatutakoa abiapuntutzat hartuta. Horretarako, lehenik eta behin, Bildu GFAaren gobernura heldu zeneko testuingurua eta BZtik egin zen proposamen politikoaren marko kontzeptuala aztertuko ditugu. Ondoren, erakundeen birasmatzerako gakoetako batzuk aztertzen dira. Eta, azkenik, ikasgai batzuk ateratzen dira, hasi diren trantsizio prozesuen sendotzerako argibide izan nahi dutenak.

Hitz gakoak: bizitzaren iraunkortasuna, pedagogia, erakunde publikoak, trantsizioa, feminismoa. 


\section{Introducción}

Me cuentan que cuando se lanza una piedra y esta golpea una herida, las reacciones primarias son el ataque, la huida o la parálisis. Que el deseo siempre nace del dolor. Y que abrazar el dolor para entenderlo es abrazar la posibilidad de poner en marcha el mecanismo de transformación de este en un anhelo que impida su repetición. Se trataría de rescatar para lo común un sentido ético superior que anhele un vivir bien entre nosotrxs ${ }^{1}$, contigo y conmigo, que atenúe el dolor, individual y colectivo, y rescate la reciprocidad como el principio esencial que garantiza nuestra supervivencia. Un anhelo bastante distinto al deseo de vivir mejor individualista y separado de lxs demás, a expensas de las demás y de la naturaleza. Desde esta doble perspectiva, la necesidad de otrxs para sobrevivir y rescatar de la condición humana aquello que atenúa el dolor, parece sensato crear espacios y formas colectivas cuyo anhelo esté al servicio de ese fin.

La precarización de la vida de la gente y la multiplicación de las desigualdades sociales parecen un buen indicador para medir que no lo estamos haciendo muy bien. Señala Amaia P. Orozco (Orozco, 2014:22)

Dicen, desde el ecologismo social y el movimiento por el decrecimiento, que el mundo está cambiando, es más, que el cambio es insoslayable, va a ser sí o sí, por lo que la pregunta no es si podemos evitarlo o frenarlo. La pregunta es si queremos gobernarlo con criterios de justicia o dejamos que se gobierne con criterios de mercado.

Si el cambio es ya, en el tránsito hacia otros mundos posibles no queda otra que tirar de memoria y aprender de los errores, y entender que las utopías se

\footnotetext{
${ }^{1}$ En este artículo optamos por el uso de expresiones genéricas, por la utilización del femenino y el masculino, así como por la desobediencia lingüistica, haciendo uso de la $x$, en aquellos casos donde la primera no es posible o para emplear una fórmula más inclusiva en términos de identidades sexuales y de género. El objetivo no es otro que visibilizar y abrir procesos de ruptura con la normatividad de una lengua que expresa y reproduce las relaciones de poder entre mujeres y hombres. Tal y como señala Amaia P. Orozco (2014:33): «convenzámonos de que la transgresión lingüística no es peligrosa para la salud, de que la salud de la propia lengua no se pone en peligro por el cambio y desobedezcamos».
} 
construyen desde las prácticas concretas y exigen arremangarse y embarrarse en el fango de la descomposición de lo inhumano y las dificultades de inventar con lo mejor del ser humano. Porque el fracaso nos informa de nuestra imperfección y fragilidad pero, también, de nuestra capacidad de recuperarnos y hacernos más fuertes.

Estas páginas nacen de una experiencia de gobierno fracasada al menos en dos sentidos: perder el apoyo electoral de la mayoría social y, con ello, la imposibilidad de consolidar los procesos de cambio iniciados. Y nacen de un anhelo: que lo hecho, disfrutado, aprendido, transformado y llorado sirva para alimentar esa caja de herramientas que nos permitan perfilar certidumbres en un tránsito inevitablemente plagado de incertidumbres.

\section{Releyendo la crisis en la que llega Bildu al gobierno de la DFG}

Se trata aquí de retratar solo algunos aspectos del contexto de crisis en el que llega Bildu al gobierno de la DFG, las lecturas de esta que se hicieron desde la DI y que fueron la base sobre la que se articuló su programa político y algunas de las claves que apuntan algunas de las dificultades y limitaciones que va a tener Bildu para colocar la estrategia y la agenda feminista emancipadora en la centralidad de su acción de gobierno.

Cuando a finales de la década de los ańos 2000 estalla la crisis financiera, la lectura oficial mayoritaria del entramado institucional-político de nuestro entorno — vasco, español y europeo- hablaba de una crisis temporal que estaba generando problemas en los circuitos de la economía monetizada y entendía que con la aplicación de determinadas políticas de ajuste, que implicaban la socialización del desastre generado por la ambición sin límite de los principales agentes del mercado neoliberal, íbamos a volver a retomar la misma senda de crecimiento económico, empleabilidad y bienestar colectivo. En 2011, sin embargo, en las calles ya se oían con fuerza las voces de una multitud que se negaba a aceptar que se la apuntara como responsable de una crisis que le hablaba al oído y le susurraba que había vivido por encima de sus posibilidades y que ahora tocaba ajustarse el cinturón. Y empezaban a articular otro relato de la crisis que cuestionaba la narración oficial de sus porqués y por tanto, sus respuestas para hacerle frente. La calle empezaba a apuntar que la crisis estaba vinculada con problemas de fondo.

En este marco las voces feministas, a la par que otras miradas críticas, eran y son lapidarias. Señalan que si miramos la crisis desde la óptica de hacer la vida posible en condiciones dignas para todas las personas, esta es anterior al estallido financiero de 2008. Hablan de una crisis de modelo que comprende, al menos, tres dimensiones: 
a) La quiebra de los procesos vitales y del entorno que las sustenta por una cuádruple crisis:

- La de los cuidados en tanto en cuanto son esa cuestión que se resuelve como buenamente se pueda. La fuente principal de provisión de cuidados son los hogares, las redes de apoyo informal de estos y un trabajo doméstico que, a su vez, es una maquinaria reproductora de las desigualdades sociales y de género porque es un trabajo que algunos grupos sociales compran a otros que no pueden elegir. La respuesta desde lo público, por otra parte, se caracteriza por su escasez, el refuerzo de que las respuestas procedan de los hogares (corte familiarista) y la apuesta porque su mercantilización sea más un nuevo nicho rentable de negocio (gestión mercantil de los recursos de cuidado) que un derecho a garantizar. Ante la falta de respuestas sociales, lo que observamos es que el trabajo de cuidados está feminizado, invisibilizado y carece del reconocimiento social del aporte que hace al conjunto del sistema. En definitiva, nos encontramos con que el cuidado hoy de las personas es insatisfactorio, insuficiente, precario y no libremente elegido por quienes los siguen asumiendo, las mujeres.

— La de la reproducción social. Todos los indicadores muestran que la vida de la gente se está precarizando. Esto es, que cada vez hay más personas que no cuentan con los recursos suficientes para cubrir sus expectativas materiales y vitales. Y que, en este marco, las brechas de género inter- e intra-género aumentan. Si el grueso de las mujeres tienen más dificultades para contar con ingresos que les permitan vivir vidas autónomas, en el caso de las mujeres extranjeras y de los hogares encabezados por mujeres, la situación es aún más fea. Frente a esta situación de privación de recursos, no es aventurado señalar que muchas de ellas están recurriendo a los refugios laborales de urgencia para momentos de crisis como el empleo de hogar, el trabajo sexual o formas varias de mercantilización del cuerpo de las mujeres. Sin embargo, si miramos todos los trabajos que permiten satisfacer necesidades y no solo los que se hacen a cambio de una remuneración, se descubre que la inmensa mayoría de la población tiene algún tipo de actividad laboral. Y frente a la idea de que el conjunto social depende del sector que está en el mercado, lo que se observa es que hay un montón de bienes y servicios que se producen para satisfacer necesidades que se usan sin reconocerlos (trabajos de cuidados). Y que su producción gratuita para el conjunto coloca a las mujeres en una situación de dependencia material y precariedad vital.

— La de la salud que provoca que los cuerpos enfermen al ponerlos al servicio de la búsqueda de beneficios económicos y al desarrollar una 
mirada médica que invisibiliza ciertas dolencias y tiende a su medicalización.

- La ecológica derivada de un modelo productivo que no tiene en cuenta los límites de la corteza terrestre y está provocando el cambio climático, el agotamiento de los recursos o el colapso de la biodiversidad. La lógica productivista y sus sujetos centrales, las transnacionales, no solo están arrasando con los procesos de la tierra como base material de nuestra supervivencia, si no que sus prácticas guiadas por el ánimo de lucro constituyen armas de destrucción masiva donde se pueden producir transgénicos contaminando las semillas naturales, usando el hambre como arma de guerra o privatizando un bien público no estatal como el agua.

b) El cuestionamiento de las estructuras de representación política. Asistimos ya no a un desmantelamiento de las instituciones públicas, sino a la utilización de estas como instrumentos al servicio de la lógica de acumulación de los mercados. Vivimos, como señala Santos (2006), en democracias de baja intensidad, formalmente democráticas y socialmente fascistas, donde la idea del gobierno por el pueblo y para el pueblo es ignorada de manera indecente.

c) El cuestionamiento del sistema ético y de valores. Frente al «sálvese quien pueda», necesitamos urgentemente un nuevo contrato social, que aborde el invisibilizado contrato sexual, guiado por principios éticos que no legitimen la desigualdad y rechace cualquier asignación de los recursos que impliquen la explotación, la desprotección de las personas, su empobrecimiento o su exclusión.

Todo esto apunta a lo que algunxs han llamado segunda ruptura en el desarrollo del bienestar de Europa. En el caso del Estado español, se trataría de la primera ya que su construcción y desarrollo coincide con la primera reestructuración de los Estados de bienestar europeos derivadas de la crisis del modelo keynesiano-fordista a partir de los años 70. Un modelo profundamente patriarcal en la medida en que se fundamenta en un pacto entre el capital y el trabajo asalariado $^{2}$.

2 "Los sistemas de seguridad social, figura principal del Estado de bienestar en Europa, son emanación y soporte de la sociedad industrial. Nacen en el siglo XXI con la revolución industrial y su correlato social: la emergencia del salariado. Destinados a garantizar la continuidad de ingresos de los obreros que han perdido las redes de solidaridad familiares y locales de la sociedad agrícola, permiten al mismo tiempo a los patronos asegurarse la fidelidad, la estabilidad y la calidad de su mano de obra". "La sociedad industrial define un nuevo reparto de las tareas, en el que el hombre garantiza los ingresos y la protección social del resto de la familia, los niños están cada vez más escolarizados, y las mujeres se presupone que se quedarán en casa y se harán cargo de las tareas domésticas» Esping-Andersen y Palier (2010: 8-9) 
El Estado de bienestar español caracterizado por una protección social mixta: programas de tipo universal — sanidad o educación-, elementos corporativos-conservadores (niveles contributivos de pensiones o prestaciones por desempleo) y políticas de tipo asistencial (prestaciones no contributivas, rentas mínimas de inserción, servicios sociales, desregulación laboral creciente, etc.) y que no persigue la igualdad como objetivo, se estaría deslizando en esta crisis hacia una creciente asistencialización y mercantilización de la política social ${ }^{3}$. La falta de protección social de cada vez mayor parte de la población se ancla en la idea de que esta ya no es productiva, sino un coste, en tanto en cuanto ya no sirve para apoyar la lógica económica de acumulación. Si las instituciones se retiran para ponerse al servicio de la lógica acumulativa, necesariamente son los hogares y dentro de ellos, las mujeres, quienes asumen la responsabilidad de sacar la vida adelante.

En este marco, las voces feministas denuncian que la estrategia del sistema pasa por invisibilizar el conflicto irresoluble entre el capital y la vida para lograr la obediencia que permita hacer legítima una sociedad donde la vida está amenazada. Cuando la seducción del sistema no es suficiente para mantener el statu quo recurre al uso de la violencia. De manera preventiva, se utilizan los instrumentos legales y de política pública al servicio de mantener la situación bajo control. Es aquí donde se enmarca la reforma del artículo 135 de la Constitución, la llamada «ley mordaza» o los intentos de reforma de la ley del aborto, entre otras.

Frente a la nueva configuración socio-económica, se propone poner la sostenibilidad de la vida en el centro de las prioridades sociales. Y señalan que si se trata de enfrentar el conflicto irresoluble capital-vida, ya no se trata de elegir entre lo que teníamos, un Estado de bienestar reducido en una sociedad de mercado que se sostiene sobre la depredación medioambiental, la división sexual del trabajo y el expolio de los países de la periferia, y lo peor que estaba asomando, el Estado mínimo al servicio de una sociedad de mercado salvaje.

En este contexto de crisis sistémica que está cambiando el mundo conocido y donde las soluciones que pasen solo por la recuperación de los Estados de bienestar tal y como los conocíamos se demuestran ya no solo limitadas si no inviables. ¿Qué estaba pasando, además, en 2011 en Hego Euskal Herria?

\footnotetext{
3 «Podemos agrupar los sistemas de protección social en tres grandes familias o regímenes (el régimen socialdemócrata de los paises escandinavos, el régimen liberal de los paises anglosajones y el régimen conservador-corporativista de los paises de la Europa continental), diferenciando al mismo tiempo los objetivos politicos y sociales que tratan de alcanzar (respectivamente: la igualdad entre los ciudadanos, la simple cobertura social de los más pobres, el mantenimiento de los ingresos de los trabajadores) y los instrumentos que utilizan a tal efecto (respectivamente: politicas universales y servicios sociales gratuitos, politicas sociales dirigidas a un sector de la población restringido, seguros sociales financiados por cotizaciones sociales)" Esping-Andersen y Palier (2010: 9).
} 
Que por primera vez, Bildu, una coalición de partidos soberanistas de izquierda, aunaba esfuerzos y se comprometía a acompañarse en el tránsito hacia un nuevo escenario político, en un contexto de cese definitivo de la acción armada de ETA, que exigía generosidad y solidaridad compartida para enfrentar los intentos de la institucionalidad y la legalidad existente para frenar cualquier posibilidad de cambio. Bildu irrumpía, de este modo, en las elecciones forales y locales como segunda fuerza política a poco más de 50.000 votos del PNV y como primera fuerza en el territorio de Gipuzkoa. Por primera vez, en las últimas tres décadas de democracia representativa, el PNV perdía su hegemonía institucional en este territorio y perdía el gobierno de la Diputación de Gipuzkoa y de buena parte de sus instituciones locales.

Cuatro años después nada parece haber cambiado. Las elecciones forales y locales de mayo de 2015 instalan al PNV en alianza con el PSE, de nuevo, con inusitada fuerza en los gobiernos institucionales de los tres territorios y gana buena parte de las instituciones locales perdidas, incluso aquellas, en otro tiempo, más difícilmente disputables. Y, sin embargo, todo ha cambiado. Podemos, nuevo actor político de izquierdas articulado a nivel estatal y surgido en 2014, se presenta como un actor político que va a situarse como tercera fuerza en votos en las mencionadas elecciones y como primera fuerza en las estatales del 20 de diciembre de ese mismo año.

Si este es el relato de los hechos, una aproximación sencilla a la interpretación de estos nos señalan que Bildu no ha sido la herramienta que ha conseguido articular en torno al dolor colectivo que la crisis está provocando una respuesta social que hiciera irreversible el cambio político. Es cierto que los ecos de la crisis económica y política está teniendo efectos más moderados en Hego Euskal Herria que en el conjunto del Estado español y también que el PNV ha reforzado su hegemonía institucional en un contexto de crisis. Tal y como señala Fernández (2015b)

Los porcentajes de desempleo, pobreza o recortes en servicios sociales son muy significativos, pero no se han convertido para la mayoría de la población en una deslegitimación profunda del modelo, como sí ha podido constatarse en mayor medida en el Reino de España. De esta manera, el PNV no solo no ha sufrido desgaste electoral sino que ha reforzado su hegemonía institucional en medio de la crisis, logrando derivar el desencanto sobre la actual situación al gobierno central e incluso a autoridades y gobiernos europeos.

Las declaraciones recientes de Pedro Azpiazu, diputado del PNV en el Congreso, son ilustrativas (Aizpuru, 2015): «El PP ha abaratado el despido y ha traido sueldos indignos siguiendo la politica de recortes impuesta por Merkeli.

Pero, también, Podemos irrumpe como un actor en Hego Euskal Herria que representa para una amplia mayoría los anhelos de cambio social tanto aquí como en el Estado. 
Con este retrato del estado de cosas, no intento buscar las claves que lo explican, sino más bien situar el contexto en el que voy a enmarcar algunas de las reflexiones que pueden apuntar a comprender qué es lo que ha dificultado que la estrategia de Bildu esté al servicio de articular procesos que coloquen y aterricen una agenda feminista emancipadora en la centralidad de su acción de gobierno durante el periodo 2011-2015 y que perfilen los desafíos, que se irán señalando en este artículo, que enfrenta cualquier fuerza política que aspire a disputar y gobernar las instituciones para enfrentar el conflicto capital-vida.

Siguiendo, de nuevo, las tesis de Fernández (2015b) haré mención al menos a cuatro elementos. El primero está referido al hecho de que Bildu sea una coalición de partidos con enfoques ideológicos distintos que van desde "Enfoques socialdemócratas de clara vocación institucional, hasta apuestas anticapitalistas de confrontación con el sistema vigente en todas sus dimensiones».

El segundo y vinculado con este primero está referido a la dificultad de construir desde esta diversidad una agenda común. Comparto, en ese sentido, las tesis que señalan que la agenda se centró en dos cuestiones prioritarias, formuladas en términos muy vagos, la soberanía como medio para el cambio social y la apuesta por la resolución del conflicto vasco. Una vaguedad que permite articular un discurso que combina la crítica sistémica al modelo democrático y socio-económico y un aterrizaje programático que dibuja el hacia dónde en términos de recuperación de un Estado del bienestar adelgazado y por tanto, de retorno a un modelo capitalista frenado en sus excesos. Y esto se explica por un tercer elemento, la estrategia política de Bildu centrada en el asalto institucional y que supedita toda su actuación a la primacía de lo electoral. Ya no se trataría de articular una estrategia política que desde el aquí y ahora mire lejos en clave de transición y disputa de espacios, entre ellos las instituciones, sino en asumir la perspectiva, demostrada errónea, de que ganar las instituciones es ganar el poder. En ese empeńo, antes y después de llegar a las instituciones, se renunciaría a las propuestas que dañen o se entienda que no aportan masa electoral, al objetivo prioritario de llegar o mantenerse en las instituciones. De ahí que tras la pérdida de apoyo electoral en las elecciones de mayo de 2015, los análisis de la coalición apuntaran a señalar que se había errado al hacer los cambios tan rápidamente. Y por último, en este marco de primacía de lo electoral, la articulación de alianzas con los movimientos sociales se circunscriben más a momentos puntuales y de apoyo mutuo, según las necesidades coyunturales, que a un tejer estrategia y acción conjunta para dibujar un proceso de transición.

Todo ello creo que nos da pistas para entender tres cuestiones de su hacer institucional entre 2011 y 2015: en primer lugar, que algunas cuestiones de la agenda quedaran eclipsadas o, simplemente, no estuvieran y se fueran desarrollando posteriormente desde marcos de acción contradictorios, a veces emancipadores, a veces, social-demócratas o social-liberales, en explícita pugna para 
avanzar en los primeros. En segundo lugar, como consecuencia de lo primero, que se hiciera una comunicación miope que no permitió construir un relato y una agenda con otros agentes de cambio, proyectar un imaginario común integral y coherente y acompañar las prácticas que lo aterrizaran con argumentos pedagógicos que permitieran a la gente combatir los relatos que se esforzaban con eficacia en construir quienes no quieren que nada cambie. Y, en tercer lugar, que las respuestas del gobierno a las demandas y expectativas de la gente no se cumplieran.

Si desde el marco de la democracia representativa las elecciones son la expresión formal de la legitimidad política, este genera un doble problema: por una parte, que lxs representadxs crean que votando ya han cumplido y esperen ver satisfechas las demandas que hicieron al votar sin deliberación previa que les permita conocer las demandas de lxs otrxs, incluidas las que votaron lo mismo que ellas. Además, los grupos corporativos y organizados son los únicos capaces de abrir un conflicto social mediáticamente visible llamando la atención de las instituciones, frente a la gente no organizada cuyas demandas es probable que no sean objeto de atención. Y por otra parte, que lxs representantes se sientan autorizadxs para poner en marcha sus programas, más o menos vagos, sin articular un diálogo con lxs primeros. Además, en un contexto donde tienen información que no tienen estxs y les permite valorar las consecuencias de sus medidas.

¿Cómo solventar dichos problemas? Siguiendo a Enrique Gil (2010)

La mejor respuesta es devolver la voz al pueblo para que recupere sus canales de comunicación directa con el poder. Es la bien conocida solución de la democracia deliberativa y la democracia participativa, que exigen organizar a los ciudadanos atomizados para darles voz (deliberativa) y voto (participativo), suscitando su más activa y pluralista movilización cívica para que se constituyan en sujetos colectivos capaces de formular sus demandas al poder.

Desde esta perspectiva, podemos aventurar alguna hipótesis: que la acción de gobierno de Bildu se esforzó en dar voz a la gente, a través de procesos que facilitaron compartir sus diversas expectativas en el diálogo colectivo, como el proceso de presupuestos forales participativos, el apoyo rotundo a la organización sectorial de demandas ciudadanas (como el de las cuidadoras profesionales de las residencias y centros de día), o la respuesta a demandas latentes pero no organizadas (la Ayuda de Garantía de Ingresos para quien no tiene sus necesidades materiales más básicas cubiertas, por ejemplo). Pero que, sin embargo, al grueso de la acción de gobierno se caracterizó por la aplicación de su programa sin deliberación suficiente con la gente (en la recogida y gestión de las basuras, por ejemplo) o sin la creación de espacios de diálogo permanentes con esta que facilitaran contrarrestar los conflictos mediáticos que orquestaban los grupos de interés como Adegi (patronal guipuzcoana) apoyados por algunas fuerzas políticas de la oposición. 
En ese sentido, y siguiendo la hipótesis, una apuesta clara para que las políticas públicas participativas permearan toda la acción de gobierno, y no solo prácticas más o menos puntuales, movilizando recursos suficientes para que así fuera, hubieran podido contrarrestar los problemas que genera la democracia representativa delegativa y que, a su vez, se hubiera podido traducir en la construcción de una legitimidad de mayor calidad democrática de la acción del gobierno.

\section{Algunas claves para redefinir el papel de las instituciones en el conflicto capital-vida}

Si, como hemos visto, la estrategia del sistema pasa por invisibilizar el conflicto entre los procesos de acumulación y los procesos que sostienen la vida, la estrategia emancipadora solo puede pasar por visibilizarlo y disputar espacios y recursos a los primeros para ponerlos al servicio de los segundos. Desde esta perspectiva, surgen mil preguntas ¿Qué es apostar por los procesos que sostienen la vida? ¿Qué papel deben tener las instituciones públicas? ¿Qué pueden hacer las políticas públicas? ¿Cómo hacer la disputa? ¿Con quién pueden caminar de la mano para hacerlo?

En el aquí y ahora, la vida cada vez más precaria e invivible para la mayoría social se está resolviendo en términos de explotación, en primer lugar desde los hogares, en segundo lugar desde el mercado y, en último lugar, desde las instituciones públicas cuando se produce un fallo en la respuesta de las otras dos esferas. Si el horizonte que se propone pretende colocar el cuidado de la vida en el centro parece que es prudente reivindicar con fuerza que estas últimas tienen mucho que hacer y deben hacer.

En ese sentido, las instituciones públicas tienen que hacer movimientos que pasen por re-equilibrar la corresponsabilidad en el cuidado de la vida de la triada formada por instituciones públicas, mercado y hogares. Estas tienen un papel clave para hacerlo pero, también, para fijar cómo hacerlo para resistir y transformar las lógicas privatizadoras en los hogares o mercantilizadoras de los mercados.

Pero si se queda aquí el debate tal y como señala Amaia Pérez Orozco (2014:250) surgen varios problemas. En primer lugar que se siga haciendo desde "Una cierta noción de desarrollo como expansión de la producción, basada en la depredación ambiental y en la invisibilización de esferas y agentes", que permiten la reproducción de la vida. En ese sentido, un mayor protagonismo institucional no implica una verdadera asunción de la responsabilidad colectiva de sostener la vida y seguirá ocultando dónde y quién la está resolviendo. En segundo lugar, que las nuevas esferas de la vida que son asumidas institucionalmente lo 
hagan desde una universalidad que desprecia el principio de singularidad, esto es, el respeto a la diversidad de experiencias y condiciones vitales. Y, en tercer lugar, que se entienda la institución en términos centralizadores y dirigistas, esto es para la gente pero sin la gente.

Conviene, por tanto, alertar que si la invisibilización de las esferas socioeconómicas donde se resuelve la vida (los cuidados) no es objeto de un debate serio o es un capítulo adicional de un programa político, se estará regulando el conflicto capital-vida pero no se estará facilitando, desde las instituciones públicas, el tránsito hacia otro modelo que tenga como único fin el sostenimiento de la vida.

Si se quiere no solo resistir y regular, sino también transitar desde las instituciones, las prácticas desde las políticas públicas debieran pasar, al menos, en primer lugar, por desprivatizar los cuidados, sacarlos de los hogares asumiéndolos públicamente y no permitir que la lógica mercantil funcione en esta dimensión. En segundo lugar, por redistribuir todos los trabajos, entendiendo como trabajo tanto el remunerado como el no remunerado, lo que permitiría desfeminizar los cuidados y descentrar los mercados como espacios únicos de provisión de recursos para el bienestar. En tercer lugar, visibilizar y revalorizar, en términos sociales y económicos, todos los trabajos en función de su contribución al sostenimiento de la vida y su ajuste a los límites físicos del planeta y no apoyar y penalizar públicamente aquellos que atentan contra la vida (humana y no humana). Y en cuarto lugar, garantizar el acceso a recursos, esto es, una de las bases materiales de la autonomía vital, a través de servicios públicos, prestaciones monetarias o el acceso a un salario bien mediante la redistribución de los existentes o la creación de nuevos.

Frente a la urgencia del desafío, no parece que contemos con demasiados modelos institucionales y de gestión de la cosa pública desde la izquierda, que piensen más allá de la triada en las claves señaladas.

La experiencia desde la DI nos informa que asumir la sostenibilidad de la vida como propuesta política implica abordar al menos tres cuestiones: el qué (la agenda y las prácticas), el cómo (abordar la cuestión de la representación, sus fines, principios y formas organizativas, así como redefinir su papel de actor en la disputa) y el con quién (alianzas con otros actores políticos organizados y con la gente).

\subsection{De la agenda en resistencia a la agenda en transición: el qué}

Desde esta perspectiva, se hace necesario construir una agenda de resistencia que responda a la urgencia en el aquí y ahora para frenar la precarización de la vida de la gente y de las mujeres, en particular, al tiempo que se entiende en transición primando las políticas de redistribución frente a las de crecimiento. 
Se trata de detraer recursos de la lógica de acumulación y ponerlos a funcionar en otras esferas que prioricen la vida digna y su cuidado y que, a su vez, empiecen a apuntar otro modelo socio-económico a partir de los movimientos descritos más arriba.

En ese sentido, se trata de articular un paquete de medidas que deben ponerse en marcha de manera dialogada para aumentar su eficacia y evitar, con ello, prácticas contradictorias que las invaliden o conviertan en meras políticas enfermeras. Resulta importante entender que desde la perspectiva de las políticas públicas, casi nunca nos encontraremos con medidas de resistencia y de transición puras. Digamos que hay medidas de política pública que pueden tener efectos en ambos sentidos y, que casi siempre, van a necesitar de otras para aumentar el alcance de su impacto.

Una agenda formulada en estos términos, exige en primer lugar, asumir que no hay una batería de respuestas tipo, más bien que hay un sinfín de preguntas y una "caja de herramientas» limitada para perfilar la transición. Esto es, no queda otra que navegar en la complejidad imaginando y creando al tiempo que se hace. $Y$, en segundo lugar, entender que hay que modificar de manera urgente el horizonte temporal desde el que se diseña la agenda y desde la que se gestionan los recursos públicos. Si la revolución consiste hoy en transitar rescatando aquello que es válido para la construcción de otro modelo solo podemos hacerlo en un marco temporal no legislativo y pensar la gestión de los recursos públicos en términos temporales comprometidos con la vida humana y no humana.

En ese sentido, si bien la honestidad obliga a reconocer que la acción del gobierno de Bildu en la DFG se sitúo en clave de resistencia y, en menor medida, de regulación del conflicto capital-vida y no en pensar la transición más allá de la triada, también es cierto, que algunas de las prácticas hechas estuvieron plagadas de coraje y lucidez y sirven para apuntar el hacia dónde desde una lógica procesual de cambio.

Algunas de las prácticas impulsadas desde la DI que fueron objeto de un trabajo más intensivo desde el programa político diseñado han estado referidas a la búsqueda de prestigiar y revalorizar el trabajo de cuidados profesionales y evitar que la asunción de mayores responsabilidades por parte de la institución pasara por su mercantilización — por ejemplo, la mejora de los convenios de residencias y centros de día de Gipuzkoa o el esfuerzo por regular una nueva categoría laboral distinta a la de empleada de hogar para la contratación de los cuidados a través de la Prestación económica de Asistencia personal (PEAP)—, así como a perseguir un reparto más democrático de los recursos. En este último caso, merece la pena clasificar las prácticas en tres tipos de política pública: aquellas que buscaron abrir pedagógicamente el debate social que creara las condiciones a medio plazo para convencer de la necesidad de hacernos corresponsables del cuidado de la vida - por ejemplo, el trabajo realizado en el marco de los informes 
bianuales de pobreza y exclusión social en Gipuzkoa o el simulacro de Renta Básica de ciudadanía a través de la reforma del IRPF—; aquellas que trataban de garantizar el acceso a recursos y frenar la precariedad vital de la gente atendiendo a las dimensiones de género — por ejemplo, la regulación de la Ayuda de Garantía de Ingresos (AGI), la creación de empleo en el ámbito de los cuidados fortaleciendo los servicios públicos y formas públicas autogestionadas y de empleos corresponsables con el cuidado de la vida-; y por último, aquellas que persiguieron aumentar los ingresos de las instituciones para redistribuirlos más equitativamente y guiar el comportamiento del mercado al tiempo que condicionar el acceso a recursos públicos al desarrollo de prácticas que se corresponsabilizaran del bienestar del conjunto — por ejemplo, mediante la reforma de la política fiscal y de la política de contratación y subvención pública-.

En ambos casos, estas se acompañaron, en los casos en los que fue posible llegar a acuerdos parlamentarios con los grupos políticos de la oposición, de regulaciones normativas que implicaban que la institución asumía poner coto al poder de los mercados y a resquebrajar la mercantilización de los cuidados o que estos se siguieran resolviendo de manera privada dentro de los hogares y dentro de ellos por las mujeres 4 .

\subsection{Sobre la reinvención feminista de las instituciones: el cómo}

\subsubsection{Espacios deliberativos y construcción colectiva de un sentido que merece la pena}

Decíamos más arriba que tan importante como el qué es el cómo hacen las instituciones públicas para visibilizar y enfrentar el conflicto capital-vida. Porque si se trata de responder al aquí y ahora y transitar hacia otro modelo, la construcción de una alternativa que merezca la pena para la gente solo puede construirse con la gente y para la gente. Y eso exige crear espacios de discusión democrática de los que carecemos que permitan compartir las preguntas y corresponsabilizarnos en la búsqueda de respuestas desde el esfuerzo por superar esa dualidad de pensar lo público bien como un aparato burocrático bien como autogestión.

Desde esa perspectiva, las instituciones se tienen que entender como un actor pedagógico más que facilita herramientas conceptuales que permitan emerger una conciencia crítica que dispute lo existente pero, también, a diferencia de otros agentes de cambio, tiene el papel de coordinar procesos deliberativos que permitan escuchar más y mejor a la gente para transitar los cambios acompanándolos y negociándolos con ella. Desde esta perspectiva, por ejemplo, se hubiera

\footnotetext{
${ }^{4}$ Se puede consultar el análisis detallado de algunas de las prácticas mencionadas en Gómez Hernández, Laura (2015).
} 
tratado de abrir la discusión sobre una gestión corresponsable con la corteza terrestre en la cuestión de las basuras y sin renunciar a esta, sí plantear que en clave de proceso se deben crear las condiciones que a medio y largo plazo permitan sumar a la mayoría social en dicha lógica.

Hacerlo de este modo permite que la institución haga ese otro papel de articulador de necesidades colectivas y de facilitador de la construcción de un relato colectivo guiado por otros sentidos y otros principios éticos (universalidad y singularidad). Cualquier estrategia emancipadora tiene que pasar no solo por la satisfacción de las necesidades materiales, sino también por satisfacer la necesidad de sentido (trascendencia) que tiene el ser humano. Porque lo que tiene sentido es lo que merece la pena para la gente y nos moviliza colectivamente. Y tiene sentido lo que en este mundo ha dejado de ser una preocupación: los cuidados, la alimentación, la vivienda o los tiempos para las relaciones sociales (Monedero, 2009). La vida, en definitiva 5 .

Para hacerlo, la invención y resignificación de las palabras se tornan imprescindibles para que en el diálogo colectivo se puedan redefinir esos otros sentidos de la vida en común. Las palabras deben servir para proyectar otro imaginario al tiempo que para visibilizar las relaciones de poder, confrontar y desenmascarar las palabras robadas que sirven para hacer lo contrario de lo que dicen. Es la única forma de que puedan crecer y no ser fácilmente violadas de sus significados originales (Monedero, 2009). Así, buen vivir o sostenibilidad de la vida solo pueden ser entendidas desde una crítica radical al modelo capitalista heteropatriarcal, la democracia representativa liberal o el pensamiento racional moderno, la fiscalidad podría formularse como contribución solidaria para que responda, por ejemplo, a la idea de la corresponsabilidad de cada ciudadanx para con el resto.

\footnotetext{
5 A este respecto, creo que merece la pena rescatar lo que entienden las mujeres por buen vivir y tiene sentido (trascendencia) para ellas cuando preguntamos por ello en el proceso de diseño de la política pública de igualdad en la DFG:
}

"Nosotras el buen vivir pensamos que era darles de comer a nuestros hijos, sacarlos adelante, mandarlos a estudiar. Y todo eso lo conseguimos hacer. Llegó una crisis en la que habia muchos parados y nos tocó con los hijos en el paro. Pensamos que los chavales siguieran estudiando era bueno. Nos sacrificamos, nos apretamos el cinturón. Es que ya no teníamos ni cinturón. Aprendimos a vivir con lo que ahora se dice de dejar los libros a uno y a otro, a dejarse la ropa los unos a los otros. Aprendieron nuestros hijos a ir a los mercadillos y a ir guapos con cuatro perras. Aprendieron un montón de valores y de cosas. ¿Qué es lo que pasó? Que se han encontrado en el paro con cuarenta, cuarenta y dos, cuarenta y cuatro años. Llevan como quien dice quince años trabajando. Ahora como han estado mucho tiempo en el paro, las madres que somos viudas y estamos a cero en todo, nos sacrificamos por todo. Se metieron en hipotecas. Les llegó la crisis y les pilló con hipotecas. Y las madres que seguimos siendo viudas estamos sin un duro con las pensiones que tenemos estamos con la soga al cuello y pensando en qué lio se han metido. Y nosotras somos invisibles, nunca se nos ha valorado como mujeres y si somos viudas menos porque no se nos ve por ningún lado». (Participante en el proceso de elaboración del II Plan para la Igualdad de Mujeres y Hombres en Gipuzkoa.) 
El aterrizaje del relato en una agenda y en prácticas concretas pide, a su vez, un liderazgo, no tanto carismático, si no que ayude a formular y conseguir objetivos comunes sumando a la gente. Un liderazgo que use su autoridad como medio para construir liderazgos colectivos. Que gobernar obedeciendo implica que el ejercicio del poder debe estar al servicio de fortalecer las capacidades colectivas, compartiendo y corresponsabilizando a la gente de este y con la generosidad de entender que tiene que preparar su relevo.

En esa línea, los espacios participativos se convierten en elementos constitutivos del aterrizaje porque se necesita aunar los saberes de gentes técnicas, políticas, activistas y ciudadanas y que estos se permeen y transfieran. El aterrizaje necesita de una mirada política que se apropie profundamente del conocimiento de los instrumentos de política pública (saber técnico) que permita utilizar sus contradicciones y vacíos para abrir grietas desde donde apuntar las respuestas al servicio de otros fines (saber activista) válidos para la mayoría social (saber ciudadano).

Por otra parte, si como decíamos al inicio de este apartado, el hacia dónde compartido pretende construir lo público transcendiendo la dualidad entre burocracia y autogestión, los espacios participativos y el impulso desde las instituciones de procesos autónomos o cogestionados, cobran especial relevancia. Y lo hacen porque permiten no solo la discusión colectiva pedagógica y por tanto, los cambios de conciencia, si no también reinventar las formas de hacer política al facilitar encuentros y la formación de hábitos nuevos basados en reactivar vínculos afectivos y el sentido de la responsabilidad compartida en la construcción de esas otras formas de gestión de lo común. Y eso, a su vez, permite articular y encarnar la política en todos los ámbitos de la vida y a diversos niveles: barrios, pueblos, territorio, etc. El apoyo institucional, por ejemplo, a un proyecto cogestionado entre estas y el movimiento feminista como el de la Casa de las mujeres de Donostia es un buen ejemplo de ello en la medida en que se crea un espacio cotidiano que acoge, permite tejer estrategias de resistencia y presión, al tiempo que responder a las urgencias de las mujeres en el aquí y ahora. En definitiva, acompañar los cambios de conciencia con prácticas que construyan hábitos nuevos que se materializan en prácticas guiadas por otro sentido de lo colectivo.

\subsubsection{Las alianzas como protección y contra-ofensiva}

La reinvención institucional, en todo caso, se complica si se trata de un gobierno en minoría que obliga, en ocasiones, a la prórroga presupuestaria y que hereda un endeudamiento elevado y con un amplio volumen de créditos comprometidos. En un marco, además, donde se legisla para priorizar el pago de una deuda cuestionable o la contención del déficit público al tiempo que se carecen de aliadxs en el arco parlamentario para apoyar una reforma fiscal inte- 
gral progresiva que atienda a las dimensiones de género. Y en un marco, donde los principales medios de comunicación y élites económicas y financieras se ponen al servicio de impedir cualquier giro del modelo y del sentido común utilizando cualquier "arma» que pueda debilitar o cuestionar a quienes encarnan el cambio.

En ese sentido, las alianzas entre gobiernos que quieren ser emancipadores aparecen como una necesidad no solo para socializar experiencias y compartir aprendizajes, sino también para diseñar estrategias e instrumentos comunes que permitan contrarrestar los ataques furibundos del statu quo.

Por otra parte, asumir visibilizar y disputar espacios y recursos a los procesos del capital implica cambiar el paradigma de las políticas de igualdad. Ya no era el principio de igualdad el objetivo sino el sostenimiento de la vida como aterrizaje radical de dicho principio. Este tránsito tiene que hacerse con cautela porque en ningún caso puede servir de argumento, por la izquierda, para que el diálogo con otras miradas críticas sirva para diluir la propuesta desde claves feministas en la articulación de una respuesta integral, ni para dinamitar las alianzas feministas institucionales y políticas que las feministas hemos aprendido a cuidar. Porque aunque muy diversas, sabemos que cuando nos fragmentan nos ganan. En este caso, el diálogo con las compañeras resulta prioritario y la prudencia debe ser la compańía elegida para que la confrontación nunca debilite aún más la posición de poder marginal que sistemáticamente ocupamos y ocupan las políticas de igualdad en el entramado institucional y en un contexto global de desmantelamiento de estas.

En ese sentido, el cambio de mirada y de agenda va a afectar, también, a los instrumentos de intervención diseñados desde las políticas de igualdad. Por ejemplo, los informes de evaluación de impacto de género ya no pueden seguir afirmando que un Impuesto de sociedades como el vigente en Gipuzkoa puede tener un impacto positivo en términos de género. Si ampliamos la mirada, cuestionamos el instrumento por regresivo en términos sociales y de igualdad en toda su globalidad. En ningún caso hacerlo, al hilo de lo seńalado, debe poner en cuestión el valor de lo hecho por las compañeras en la creación y diseño del instrumento.

\subsubsection{De la colonización neoliberal y patriarcal de los gobiernos institucionales: los tiempos y lógicas de la representación}

Reinventar las instituciones pasa, también, por reinventar sus lógicas neoliberales de empleo de tiempo y sus ficciones patriarcales de estar disponibles y no tener otras responsabilidades o deseos pero, también, las que mueven a quienes las representan. En los gobiernos del cambio la representación no da dinero pero envejece, enferma y, en última instancia, expulsa (especialmente a las mujeres). 
Junto con la lógica de concentración de cargos en una misma persona en un sinfín de fundaciones, empresas públicas u organismos autónomos, la asunción de un cargo público por parte de las mujeres implica, en muchas ocasiones, la asunción de ese compromiso por parte de toda su red familiar en tanto en cuanto ellas ya no van a poder emplear los mismos tiempos para el cuidado de esta, mientras que, en otras, implica el retraso de decisiones, la renuncia a desarrollar otras esferas de su vida como la maternidad o a hacer imposible formas de organización de pareja o colectiva alternativas.

En este marco, se hace urgente redefinir formas de hacer y estar en las instituciones, vivibles, diseñando estrategias que aterricen prácticas, piensen en tiempos, creen espacios y utilicen recursos al servicio de concretar en lo cotidiano ese fin último de colocar el cuidado de la vida en el centro, también, para quienes temporalmente asumen la mochila de ser cargos públicos.

\subsubsection{De la reorganización de las políticas públicas desde el enfoque feminista de la sostenibilidad de la vida}

El aterrizaje de esta propuesta hace que la política pública de igualdad no sea una política social más, ni una política sectorial con una agenda específica (que también), sino una política transversal que hace de «faro" guiando la acción del conjunto del gobierno. Debiera haber sido el plan de gobierno de la DFG pero no lo fue. En todo caso, aunque lo hubiera sido, esta condición de «faro» exige que tenga una posición simbólica, funcional, material y de personal técnico especializado propia que le dote de la autoridad para hacerlo ${ }^{6}$.

Por otra parte, una propuesta así exige cambiarlo todo. Es un planteamiento que dinamita el sentido común de las instituciones: su fin último, sus valores, sus prioridades, cómo ingresa y cómo redistribuye el gasto público, la estructura organizativa jerárquica y estanca, así como el modelo y la organización del funcionariado público para ponerlo al servicio de un nuevo sentido.

\footnotetext{
${ }^{6}$ Así, por ejemplo, se creó una DI única colocada en el lugar simbólico y funcional más importante de la DFG: el Gabinete del Diputado General; se pasó de un presupuesto del 0,06\% a un presupuesto del 0,2\%; se aumentó el personal técnico al servicio de la Dirección, pasando de dos personas técnicas especializadas a cinco; y se elaboró y aprobó, en el parlamento territorial, la Norma Foral 2/2015, de 9 de marzo, para la Igualdad de mujeres y hombres que elevaba a rango legal no solo el enfoque feminista de la sostenibilidad de la vida sino que establecía el compromiso de la DFG para crear las condiciones estructurales para que la política de igualdad pudiera guiar desde éste al conjunto de la institución. Así, entre otras cosas, asumía la necesidad de alcanzar el 1\% del presupuesto para las políticas de igualdad en un marco temporal de seis años (2021); la necesidad de fortalecer la DI con nuevo personal especializado y la creación de unidades para la igualdad dentro de cada uno de los departamentos de la DFG con personal especializado en materia de igualdad; o establecía que la política de igualdad sería una dirección única situada en el Gabinete del Diputado General.
} 
Desde la perspectiva procesual de urgencias y tránsito, la agenda debiera empeñarse en las políticas públicas con más capacidad para transformar las estructuras que producen y reproducen las desigualdades sociales y de género como la política fiscal, la política de cuidados, las políticas que facilitan el acceso a recursos, las políticas con capacidad de poner límites y coto a la lógica depredadora de los mercados y, a su vez, vincular el análisis de cómo interactúan los impuestos y los gastos con un modelo de democracia de alta intensidad.

\subsubsection{De las habitaciones propias al diálogo con otros agentes sociales de cambio y con las que nunca están: el con quién}

Hacerse eco desde la institución de las preguntas y análisis sobre la crisis que estaba haciendo el movimiento feminista y otros agentes sociales de cambio implica entender que las alianzas con esxs otrxs no son solo una cuestión de deseo o justicia, sino de necesidad, si se quiere construir una agenda común para que los esfuerzos de cada cual remen sumando y multipliquen los ecos de otro sentido.

La construcción de esa agenda desde la interseccionalidad de miradas exige crear y fortalecer los espacios feministas como condición. Y esto implica, reconocer de dónde se parte, darles voz colocando a la institución, en muchas ocasiones, en un segundo plano y dotarles de recursos que les facilite hacerlo. Entre otros, facilitando la transferencia de conocimientos que alimente la discusión y que puedan ser devueltos en forma de propuesta para volver a ser dialogada.

Pero hacerlo está sembrado de complejidad. La institución no puede olvidar que la calle organizada en alianza es un lugar deliberativo, propositivo y un lugar desde el que inventar otras formas de gestionar lo común pero, también, desde el que tensionarla y vigilarla. Por otra parte, la calle organizada se enfrenta a la tarea de pasar de una agenda de la confrontación a una agenda común de construcción con instituciones viejas que quieren transformarse para ponerse al servicio de otras cosas. Para hacerlo, parece inevitable que tengan que abrir en sus entrañas el debate honesto sobre sus fuerzas reales, su agenda política, sus estrategias de incidencia y de acción colectiva.

Algunos elementos que permiten ir solventando dicha complejidad pasan en primer lugar por articular un diálogo honesto y transparente que entienda que la lealtad de las que, temporalmente, estamos dentro no es hacia el partido o partidos en el gobierno (se combate de este modo el patriotismo de partido y sus lógicas) sino hacia los principios que nos han llevado hasta allí y que deben guiar la estrategia y la práctica desde la institución.

Además, hacerlo de este modo, por una parte, posibilita un empatizar con los lugares que ocupa cada cual — cargos públicos, personal técnico, activistas y ciudadanía_-, al tiempo que facilita ir aclarando los papeles de cada cual en lo 
público desde la corresponsabilidad de ir tejiendo las alternativas. Esto, a su vez, permitiría evitar la temida desmovilización del movimiento feminista y otros movimientos sociales en los gobiernos de cambio en la medida en que la posición de este se entiende como un actor más en un modelo que se piensa más allá de la triada para redefinir y gestionar lo público.

Y facilita ir solventando las tensiones que se generan sobre las velocidades y profundidades de los cambios, negociando los tiempos de tránsito, y sobre las contradicciones, a veces, irreconciliables, entre la necesidad de poner en marcha medidas que respondan al ahora urgente (eficacia) al tiempo que sean el resultado de procesos deliberativos amplios.

Por ejemplo, en la redefinición de la política pública que avanzara en el derecho a vivir vidas libres de violencia machista, se logró avanzar el diálogo con el movimiento feminista acerca de la conceptualización de la violencia machista que se hizo, su aterrizaje en el marco de la institución y de las políticas públicas, el rediseño de recursos y el accesos a estos, pero no en su reconocimiento ni en la redefinición del papel de cada cual en la defensa y aterrizaje de dicho derecho.

Y de nuevo aquí topamos con otro elemento de dificultad vinculado con la tensión permanente entre los tiempos institucionales y los tiempos de la vida de la gente.

Por una parte, los procesos de construcción de agenda común exigen crear confianzas y crear condiciones estructurales que la aterricen. En nuestro caso, se hizo a través de dos procesos participativos: la elaboración del II Plan para la igualdad de mujeres y hombres y la creación y regulación de GUNEA -Espacio de participación e interlocución para la igualdad- entre el movimiento feminista y asociativo de mujeres por la igualdad y la DFG. En ambos procesos, considerados conjuntamente, se emplearon 3 años.

Por tanto, trabajar desde el enfoque relatado implica transcender la lógica política institucional de funcionamiento, en clave de legislaturas, porque si articulamos procesos de cambio que quieren hacerse con la gente, tienen que adaptarse a la lógica de funcionamiento de los tiempos de la vida de la gente. Además, no podemos perder de vista que participa no solo quien quiere porque tiene un sentido de la corresponsabilidad más elevado sino, también, quien puede. Los tiempos de vida se distribuyen de manera desigual por sexo, estatus migratorio o poder adquisitivo. Y si asumimos que la institución no debe aspirar a representar a las voces que nunca están — precarias, cuidadoras a tiempo completo, trabajadoras sexuales, migrantes internas de hogar, etc.- no solo tienen que ser creativa para articular su participación, sino que debe asumir que sin la mejora de las condiciones materiales y de tiempo de la vida de las mujeres diversas que amplíe sus márgenes de elección, va a ser imposible que alguna vez estén. 
En ese sentido, pese a los esfuerzos hechos desde la DFG, el trabajo que se hizo fue insuficiente en términos de alianza. Algunas de las batallas más importantes que habíamos ganado como la mejora de las condiciones laborales de las cuidadoras profesionales en los servicios públicos de cuidado las habíamos conseguido gracias a la alianza sindical. Lograron que las cuidadoras profesionales unidas resistieran en huelga hasta lograr los objetivos propuestos. Sin embargo, esta pelea no fue apoyada por el conjunto de las mujeres guipuzcoanas y por extensión, del conjunto social.

Por eso, crear «habitaciones» propias feministas es necesario pero, en ningún caso, suficiente. Asumir la propuesta política definida desde la DI implica crear las condiciones que permitan que las políticas de igualdad dejen de monologar entre ellas y hacia dentro de las instituciones y comiencen a dialogar con otras políticas sectoriales y hacia fuera con otros agentes de cambio y con la ciudadanía, en su conjunto.

En ese sentido, aunque apenas pudimos perfilarlo, sí contemplamos la creación de un espacio que sirviera como herramienta pedagógica al servicio de ese diálogo que facilitara la construcción conjunta entre la institución y otros agentes de cambio y que llamamos ampliar la mirada. Un instrumento que quería servir para asumir el reto de construir algo nuevo desde lo existente. Quería ser ese laboratorio de proyección de otros imaginarios posibles y de contribución a la construcción aterrizada de la transición hacia esa otra forma de organizar lo común y las vidas.

\section{Sostenibilidad y consolidación del tránsito: lecciones}

En mayo de 2011 desde la DI habíamos intuido, primero, y entendido, después, que las instituciones eran un lugar de batalla clave para disputarle espacios, recursos y sentido al capital para una vida cuyo sentido se formulaba en términos éticamente superiores. Trabajamos y nos peleamos hasta el agotamiento para que la DFG cambiara de rumbo, cambiándose a sí misma y se hiciera responsable de algunas dimensiones de lo que empezábamos ya, algunas, a definir como buen vivir. Pero los resultados de las elecciones del 25 de mayo de 2015 nos dijeron que habíamos perdido a la gente.

La herida había emergido. Podíamos señalar con el dedo responsabilidades y culpas fuera o dentro, podíamos resignarnos con los argumentos de los lugares comunes y tirar para adelante hasta la próxima (en definitiva nuestro lugar natural son los afueras), o podíamos abrazar el dolor individual y colectivo, escucharlo encarnado con atención y tratar de entender qué podíamos aprender. Porque solo aprendiendo podemos saber qué no queremos e iluminar el tránsito, desde otro lugar sí, pero más lúcido y sabio. 
Algunas de las reflexiones que siguen a continuación quieren servir, precisamente, a ese esfuerzo por cartografiar por dónde seguir a partir de las lecciones que da la práctica y la memoria. Y hemos aprendido que las alternativas se construyen desde un relato que desvela lo que tiene sentido último para la gente y transforma el dolor social y la resignación en razones que nos doten de autoridad para sońar otras vidas posibles. Una alternativa solo moviliza si merece la pena para la gente. Y lo que la moviliza pasa por construir en lo público lo que anhelamos para nuestras vidas privadas. Esto es, una vida vivida que rescate lo mejor que podemos llegar a ser. En ese marco, las instituciones públicas son un lugar de batalla y un actor pedagógico más pero no solo. Son el instrumento integral más eficaz para facilitar los cambios de conciencia y para materializar ese otro sentido en prácticas concretas. Pero también son el resultado de la correlación de fuerzas de un momento histórico (Linera, 2011). Porque tener el gobierno no es tener el poder. Y eso condiciona los ritmos y la profundidad de los cambios.

Si aspiramos a contar con instituciones que miren y actúen desde perspectivas emancipadoras, tenemos que entender que no lo harán si no se llega a ellas desde una estrategia política que las entienda como instrumentos al servicio de facilitar esos cambios de conciencia. Y para hacerlo no queda otra, antes y después de llegar a estas, que la estrategia pase por tejer alianzas en la construcción del relato y la agenda, de manera prioritaria, con el movimiento feminista y otros agentes de cambio, escuchando más y mejor a la gente para articular una respuesta que abra, ya no una grieta, sino la grieta que impida cualquier posibilidad de no cambio del sistema. Habremos, entonces, creado las condiciones para ocupar y desbordar las instituciones al tiempo que sostener el tránsito con la gente.

Pero para hacerlo, también, el sostenimiento de la vida tiene que ocupar la centralidad de esta y por tanto, entender que enfrentar el conflicto capital-vida implica no solo responder a las urgencias, ni solo resistir o regularlo, sino disputar saberes, recursos, espacios y formas de hacer al capital para ponerlos a funcionar en otra lógica desde la que se perfile la alternativa. Desde esta perspectiva, las instituciones públicas, las medidas de política pública, los espacios y formas de hacer que suman o los instrumentos de comunicación, lejos de ser un fin en sí mismo, son una caja de herramientas de las que hacer uso para ampliar las grietas e ir haciendo al tiempo que blindando el suelo por el que se camina. Y ocupar la centralidad de la agenda, también, significa que los espacios, cuerpos, políticas y recursos que deben guiar el tránsito solo pueden ser profundamente feministas. Porque entiende el heteropatriarcado como elemento constitutivo de lo que tenemos. Porque el tránsito se hace desde la memoria de nuestra genealogía feminista. Y, porque considera que lo personal es político y, por tanto, los cambios no solo provienen de los afueras (estructuras) sino, también, de nosotrxs mismxs (P. Orozco, 2014:32). 
Hacerlo de este modo, permite leer los cambios desde una perspectiva de proceso en transición. También, las derrotas electorales. Y darles el valor que tienen si a pesar de esta, se ha conseguido avanzar en la definición y recuperación de espacios en disputa desde la defensa de la sostenibilidad de la vida, en el fortalecimiento de las capacidades de los agentes de cambio, en una ciudadanía más consciente y corresponsable y aprende de los errores para afinar o, en su caso, reconstruir la estrategia. Porque, de nuevo, las victorias institucionales parciales no sostenidas sobre procesos colectivos amplios pueden apuntar la derrota de toda posibilidad de alternativa durante mucho tiempo (Fernández, 2015).

Ellas nos dan esa lucidez en la derrota:

Hoy es un día diferente para nosotras. En nuestros años de lucha hemos hecho miles de pancartas, hemos roto el cómodo silencio de los despachos oficiales, hemos hecho huelgas de casi dos años, hemos sido despedidas, hemos sido explotadas sin compasión alguna en todas las residencias, incluidas las que no tienen ánimo de lucro y hasta las religiosas. Hemos hecho de todo... pero esto no. Han pasado cuatro ańos y las cosas han cambiado. Y mucho. Por primera vez hemos comprobado que desde la política se puede apoyar a las trabajadoras, que no todos los políticos son iguales, que se puede decidir invertir en las personas en vez de mejorar el beneficio de las empresas, que se puede decidir mejorar la vida laboral de miles de mujeres. Y lo habéis hecho'.

\section{Bibliografía}

AIZPURU, Miguel (2015): «El primer debate electoral pone la economía en el centro de la agenda", Noticias de Gipuzkoa, 3 de diciembre.

DIPUTACIÓN FORAL DE GIPUZKOA (2012): II Plan para la Igualdad de Mujeres y Hombres en Gipuzkoa (2013-2020) en: http://berdintasuna.gipuzkoa.net/es/ berdintasunerako-ii.-foru-plana

DIPUTACIÓN FORAL DE GIPUZKOA (2015): Norma Foral 2/2015, de 9 de marzo, para la Igualdad de Mujeres y Hombres (BOG n. ${ }^{\circ}$ 50, 16-03-2015). En: http:// berdintasuna.gipuzkoa.net/es/impulso-coordinacion-y-seguimiento-de-las-politicassectoriales-para-la-igualdad

ESPING-ANDERSEN, Gota y PALIER, Bruno (2010): Los tres grandes retos del Estado de bienestar, Barcelona, Ariel.

FERNÁNDEZ, Gonzalo (2014): «Redefinir la política, prioridad estratégica para la izquierda», Rebelión. En: http://www.rebelion.org/noticia.php?id=18586

FERNÁNDEZ, Gonzalo (2015a): «EH Bildu y Podemos: ¿Asalto a los cielos o travesía en el desierto?, Viento Sur, 3 de septiembre. En: http://vientosur.info/spip. php?article10439

7 Fragmento de la carta de despedida enviada a Ander Rodríguez, ex-diputado del departamento de Política social y a su equipo, por todas las cuidadoras profesionales de residencias y centros de día de Gipuzkoa. 
FERNÁNDEZ, Gonzalo (2015b): «EH Bildu, el reto de la definición política», Rebelión, 21 de noviembre. En: http://www.rebelion.org/noticia.php?id=205922

FERNÁNDEZ-SAVATER, Amador (2015): "Jon Beasley-Murray: “La clave del cambio social no es la ideología, sino los cuerpos, los afectos y los hábitos"”, Rebelión, 25 de febrero. En: http://www.rebelion.org/noticia.php?id=195829

GARCÍA LINERA, Álvaro (2010): La construcción del Estado, Conferencia en la Facultad de Derecho de la Universidad de Buenos Aires, 8 de abril. En: http://www.sociales. uba.ar/wp-content/uploads/Clase-Magistral-Garc\%C3\%ADa-Linera-en-Sociales.pdf

GARCÍA LINERA, Álvaro (2011): La quinta fase del proceso de cambio: las tensiones creativas de la revolución, La Paz, Bolivia, Vicepresidencia del Estado Plurinacional de Bolivia.

GIL, Enrique (2010): "Calidad democrática y enfoque teórico y posible medición» en "Calidad democrática y Buen gobierno, Madrid, Agencia Estatal de Evaluación y Calidad, Ministerio de Política Territorial y Administración Pública, y Federación española de Municipios y Provincias.

GÓMEZ, Laura (2015): «Sostenibilidad y política para la vida cotidiana: la práctica y la lucidez de la derrota», en Galcerán, Montse; Del Re, Alisa; Briceño, Alice, Perez Orozco, Amaia; Casassas, David; Flores, Judith; Gómez, Laura; Vila, Nuria Hacia nuevas instituciones democráticas. Diferencia, sostenimiento de la vida y politicas públicas, Madrid, Traficantes de Sueños.

MONEDERO, Juan Carlos (2009): El gobierno de las palabras. Politica para tiempos de confusión, Madrid, Fondo de Cultura Económica de España, s.l.

PEREZ OROZCO, Amaia (2014): Subversión feminista de la economía. Aportes para un debate sobre el conflicto capital-vida, Madrid, Traficantes de sueños.

PÉREZ OROZCO, Amaia (2015): «Políticas al servicio de la vida: ¿políticas de transición?» en Galcerán, Montse; Del Re, Alisa; Briceño, Alice, Perez Orozco, Amaia; Casassas, David; Flores, Judith; Gómez, Laura; Vila, Nuria, "Hacia nuevas instituciones democráticas. Diferencia, sostenimiento de la vida y politicas públicas», Madrid, Traficantes de Sueños.

SANTOS, Boaventura de Sousa (2006): Renovar la teoría critica y reinventar la emancipación social, CLACSO, Buenos Aires. 